\title{
Near Real-Time Surveillance of U.S. Norovirus Outbreaks by the Norovirus Sentinel Testing and Tracking Network - United States, August 2009-July 2015
}

Minesh P. Shah ${ }^{1,2}$; Mary E. Wikswo²; Leslie Barclay²; Anita Kambhampati²,3; Kayoko Shioda ${ }^{2,3}$; Umesh D. Parashar ${ }^{2}$; Jan Vinjé2 ${ }^{2}$ Aron J. Hall ${ }^{2}$

Norovirus is the leading cause of endemic and epidemic acute gastroenteritis in the United States (1). New variant strains of norovirus GII.4 emerge every 2-4 years (2-4) and are often associated with increased disease and health care visits (5-7). Since 2009, CDC has obtained epidemiologic data on norovirus outbreaks from state health departments through the National Outbreak Reporting System (NORS) (8) and laboratory data through CaliciNet (9). NORS is a web-based platform for reporting waterborne, foodborne, and enteric disease outbreaks of all etiologies, including norovirus, to CDC. CaliciNet, a nationwide electronic surveillance system of local and state public health and regulatory agency laboratories, collects genetic sequences of norovirus strains associated with gastroenteritis outbreaks. Because these two independent reporting systems contain complementary data, integration of NORS and CaliciNet records could provide valuable public health information about norovirus outbreaks. However, reporting lags and inconsistent identification codes in NORS and CaliciNet records have been an obstacle to developing an integrated surveillance system.

In 2012, CDC launched Norovirus Sentinel Testing and Tracking (NoroSTAT), a collaborative network with selected state health departments that report specific epidemiologic and laboratory data on norovirus outbreaks to CDC via NORS and CaliciNet within 7 business days, and provide consistent identification codes for each outbreak (https://www.cdc.gov/norovirus/reporting/norostat/). The five states initially participating in NoroSTAT reduced reporting lag to NORS from a median of 22 to 2 days $(\mathrm{p}<0.001)$ and to CaliciNet from a median of 21 to 3 days $(\mathrm{p}<0.001)$. Nonparticipating states had no change in reporting lag to NORS, with a median of 26 days pre- and post-NoroSTAT implementation, and a reduction in reporting lag to CaliciNet from a median of 21 to 11 days $(\mathrm{p}<0.001)$.
CaliciNet outbreaks that were linkable to NORS outbreaks increased from $86 \%$ to $95 \%(\mathrm{p}<0.001)$ for NoroSTAT states, and from $29 \%$ to $33 \%$ ( $\mathrm{p}=0.016$ ) for other states. NoroSTAT effectively integrates epidemiologic and laboratory surveillance data to provide near real-time monitoring of norovirus outbreak activity in the United States, thereby improving public health surveillance and guiding appropriate response.

Norovirus outbreak reports in NORS and CaliciNet from the five states that participated in the first 3 years of NoroSTAT (Minnesota, Ohio, Oregon, Tennessee, and Wisconsin) were compared with all other U.S. states, Washington, DC, and Puerto Rico. Only outbreaks reported with norovirus as the single confirmed or probable disease etiology were included. All transmission modes for norovirus (person-to-person, water, food, environment, and unknown) were included. The 3-year period after NoroSTAT introduction (August 2012-July 2015, "post-NoroSTAT") was compared with the preceding 3-year period (August 2009-July 2012, "pre-NoroSTAT").

Per capita reporting rates for each state were calculated by dividing the number of outbreaks reported to NORS and
INSIDE
190 Continued Endemic Wild Poliovirus Transmission in Security-Compromised Areas - Nigeria, 2016
194 Notes from the Field: Francisella tularensis Type B Infection from a Fish Hook Injury — Minnesota, 2016
195 Announcement
197 QuickStats

Continuing Education examination available at https://www.cdc.gov/mmwr/cme/conted_info.html\#weekly.

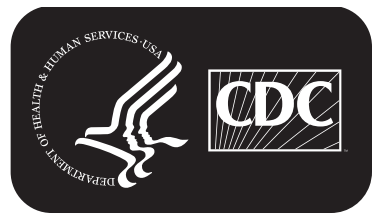

U.S. Department of Health and Human Services Centers for Disease Control and Prevention 
CaliciNet by U.S. Census intercensal yearly population estimates (10), and were expressed as outbreaks per 1,000,000 person-years. Report timeliness for NORS was assessed by determining the interval in days between the first report of an outbreak to a state health department and the date a NORS report was submitted. Report timeliness for CaliciNet was assessed as the difference in days between the date of receipt of a stool specimen at a public health laboratory and the date a CaliciNet report was submitted. The proportion of reports submitted within 7 business days was evaluated.

A complete NORS report was defined as a report that contained complete information for all fields required for NoroSTAT participation: date the first person in the outbreak became ill, primary transmission mode, exposure setting, and the number of ill persons. Linking ability was defined as the ability to link CaliciNet reports manually with a NORS report by user-submitted identification variables (automated linking was not possible during the period of analysis). The proportion of linked reports was assessed for NoroSTAT states and for other states. Norovirus genotypes reported to CaliciNet by NoroSTAT states and by other states were evaluated pre- and post-implementation of NoroSTAT. The primary transmission modes and outbreak settings reported to NORS by states participating in NoroSTAT were compared with other states and were similarly evaluated pre- and post-NoroSTAT implementation. CaliciNet-NORS linked reports were further analyzed to determine the distribution of reported transmission modes, outbreak settings, and norovirus genotypes from each reporting system. All analyses were completed using statistical software. Wilcoxon's signed-rank test was used for numerical comparisons of reporting lag and outbreak size, and chi-square tests or Fisher's exact tests were used for categorical comparisons, with a p-value $<0.05$ considered statistically significant.

The median outbreak reporting rates to NORS from NoroSTAT states before and after NoroSTAT introduction were similar (17.3 and 21.0 per $1,000,000$ person-years, respectively) (Table 1). The median reporting rate to NORS from non-NoroSTAT states increased, from 3.0 per 1,000,000 person-years (pre-NoroSTAT) to 4.1 (post-NoroSTAT). The median reporting rate to CaliciNet from NoroSTAT states increased significantly from 4.9 (pre-NoroSTAT) to 9.0 (post-NoroSTAT). The median reporting rate to CaliciNet from non-NoroSTAT states was similar pre- (2.6) and postNoroSTAT (2.1).

The median reporting interval to NORS significantly declined in NoroSTAT states from 22 to 2 days and to CaliciNet from 21 to 3 days (Table 1). No change in reporting interval to NORS occurred in non-NoroSTAT states (median = 26 days pre- and post-NoroSTAT); however, there was a significant decline in the median reporting interval to CaliciNet from 21 to 11 days. The percentage of NORS reports submitted within 7 business days increased significantly, from $26 \%$ to $95 \%$ among NoroSTAT states, and increased marginally in non-NoroSTAT states (from $12 \%$ to $13 \%$ ). The percentage of CaliciNet reports submitted within 7 business days increased from $29 \%$ to $95 \%$ among NoroSTAT states;

The MMWR series of publications is published by the Center for Surveillance, Epidemiology, and Laboratory Services, Centers for Disease Control and Prevention (CDC), U.S. Department of Health and Human Services, Atlanta, GA 30329-4027.

Suggested citation: [Author names; first three, then et al., if more than six.] [Report title]. MMWR Morb Mortal Wkly Rep 2017;66:[inclusive page numbers].

\section{Centers for Disease Control and Prevention}

Anne Schuchat, MD, Acting Director

Patricia M. Griffin, MD, Acting Associate Director for Science

Joanne Cono, MD, ScM, Director, Office of Science Quality

Chesley L. Richards, MD, MPH, Deputy Director for Public Health Scientific Services

Michael F. Iademarco, MD, MPH, Director, Center for Surveillance, Epidemiology, and Laboratory Services

\section{MMWR Editorial and Production Staff (Weekly)}

Sonja A. Rasmussen, MD, MS, Editor-in-Chief

Charlotte K. Kent, $\mathrm{PhD}$, MPH, Executive Editor Jacqueline Gindler, MD, Editor

Teresa F. Rutledge, Managing Editor

Douglas W. Weatherwax, Lead Technical Writer-Editor

Stacy A. Benton, Soumya Dunworth, PhD, Teresa M. Hood, MS, Technical Writer-Editors

Timothy F. Jones, MD, Chairman

Matthew L. Boulton, MD, MPH Virginia A. Caine, MD

Katherine Lyon Daniel, PhD

Jonathan E. Fielding, MD, MPH, MBA

David W. Fleming, MD
Martha F. Boyd, Lead Visual Information Specialist Maureen A. Leahy, Julia C. Martinroe, Stephen R. Spriggs, Tong Yang, Visual Information Specialists

Quang M. Doan, MBA, Phyllis H. King,

Terraye M. Starr, Moua Yang,

Information Technology Specialists
MMWR Editorial Board

William E. Halperin, MD, DrPH, MPH

King K. Holmes, MD, PhD

Robin Ikeda, MD, MPH

Rima F. Khabbaz, MD

Phyllis Meadows, PhD, MSN, RN

Jewel Mullen, MD, MPH, MPA
Jeff Niederdeppe, $\mathrm{PhD}$

Patricia Quinlisk, MD, MPH

Patrick L. Remington, MD, MPH

Carlos Roig, MS, MA

William L. Roper, MD, MPH

William Schaffner, MD 
Morbidity and Mortality Weekly Report

TABLE 1. Reporting indicators of norovirus outbreaks reported to the National Outbreak Reporting System (NORS) and CaliciNet — Norovirus Sentinel Testing and Tracking, United States, ${ }^{*}$ August 2009-July 2015

\begin{tabular}{|c|c|c|c|c|c|c|}
\hline \multirow[b]{2}{*}{ Reporting indicators } & \multicolumn{3}{|c|}{ NoroSTAT states $(n=5)$} & \multicolumn{3}{|c|}{ Other states $(n=47)$} \\
\hline & $\begin{array}{l}\text { Pre-NoroSTAT } \\
\text { Aug 2009- } \\
\text { Jul } 2012\end{array}$ & $\begin{array}{l}\text { Post-NoroSTAT } \\
\text { Aug 2012- } \\
\text { Jul } 2015\end{array}$ & p-value & $\begin{array}{l}\text { Pre-NoroSTAT } \\
\text { Aug 2009- } \\
\text { Jul } 2012\end{array}$ & $\begin{array}{l}\text { Post-NoroSTAT } \\
\text { Aug 2012- } \\
\text { Jul } 2015\end{array}$ & p-value \\
\hline Total NORS reports & 1,357 & 1,981 & - & 2,843 & 3,738 & - \\
\hline Median NORS reports per $1,000,000$ p-y* (range) & $17.3(4.1-32.8)$ & $21.0(6.1-41.5)$ & 0.16 & $3.0(0.1-55.1)$ & $4.1(0.1-72.2)$ & 0.045 \\
\hline Median reporting lag (days) & 22 & 2 & $<0.001$ & 26 & 26 & 0.29 \\
\hline No. (\%) reported within 7 business days & $359(26)$ & $1,888(95)$ & $<0.001$ & $328(12)$ & $500(13)$ & 0.026 \\
\hline No. (\%) with all required fields completed & $1,183(87)$ & $1,979(99.9)$ & $<0.001$ & $1,235(43)$ & $2,396(64)$ & $<0.001$ \\
\hline Total CaliciNet reports & 657 & 1,077 & - & 1,885 & 2,174 & - \\
\hline Median CaliciNet reports per 1,000,000 p-y (range) & $4.9(1.9-22.0)$ & $9.0(2.6-26.2)$ & 0.036 & $2.6(0.05-34.6)$ & $2.1(0.08-24.7)$ & 0.63 \\
\hline Median reporting lag (days) & 21 & 3 & $<0.001$ & 21 & 11 & $<0.001$ \\
\hline No. (\%) reported within 7 business days & $188(29)$ & $1,018(95)$ & $<0.001$ & $196(10)$ & $651(30)$ & $<0.001$ \\
\hline $\begin{array}{l}\text { No. (\%) linkable to NORS reports with } \\
\text { reporter-supplied ID }\end{array}$ & $564(86)$ & $1,027(95)$ & $<0.001$ & $552(29)$ & 718 (33) & 0.016 \\
\hline
\end{tabular}

Abbreviations: NoroSTAT = Norovirus Sentinel Testing and Tracking; $p$-y = person-years.

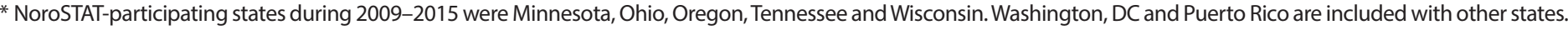

a more modest, but still significant increase (10\% to 30\%) occurred among non-NoroSTAT states.

NORS reports with all NoroSTAT-required fields completed increased significantly, from $87 \%$ to $99.9 \%$ among NoroSTAT states, and from $43 \%$ to $64 \%$ in non-NoroSTAT states. The percentage of CaliciNet reports that were linkable to NORS reports also increased significantly among both NoroSTAT states (from $86 \%$ to $95 \%$ ) and all other states (from $29 \%$ to $33 \%)$. Over the entire 6-year period, 2,861 CaliciNet reports linked to NORS reports across all states, providing more complete data on the outbreaks than either system alone. The NORS reports provided the transmission mode for all 1,106 (100\%) linked CaliciNet records without an identified transmission mode, and the outbreak setting for 669 (60\%) CaliciNet records without an identified setting. In addition, CaliciNet reports provided the genotype for all 719 linked NORS records without a reported genotype.

The genotypes of norovirus outbreaks reported to CaliciNet by NoroSTAT states were similar to those reported from nonNoroSTAT states (Table 2). In the pre-NoroSTAT period, GII.4 New Orleans was the dominant genotype in both NoroSTAT and non-NoroSTAT states, accounting for 54\% and $65 \%$ of outbreaks in these states, respectively. The dominant genotype shifted to GII.4 Sydney in the post-NoroSTAT period in both NoroSTAT and non-NoroSTAT states, accounting for $61 \%$ and $68 \%$ of outbreaks, respectively. Similarly, the transmission modes and outbreak settings reported to NORS by NoroSTAT states were representative of national data, both pre- and post-NoroSTAT implementation (Table 2). Personto-person transmission was the predominant transmission mode, and long-term care facilities were the most common outbreak setting.

\section{Discussion}

Substantial improvements in norovirus outbreak reporting, measured by the volume, timeliness, and completeness of epidemiologic and laboratory reports have been observed since the introduction of NoroSTAT in participating states, likely because of stringent reporting requirements and enhanced communication between epidemiologists and laboratorians in both state health departments and at CDC. NoroSTAT participating states are providing near real-time norovirus outbreak surveillance data to CDC, with $95 \%$ of NORS and CaliciNet reports submitted by these states within 7 business days. States not participating in the NoroSTAT network saw more modest improvements in report timeliness and completeness, likely because of general improvements in the report submission user interface, and increased engagement of state health departments and laboratories with annual meetings, workshops, and newsletters.

Ninety-five percent of CaliciNet reports submitted by NoroSTAT states were linked to NORS reports, fostering better integration and coordination of epidemiologic and laboratory data. These linked reports provide more complete and accurate reporting of norovirus outbreaks, and their value is illustrated by the large proportion of reports for which data from one system supplement those from the other. Report linkages were completed manually and retrospectively during the period of this analysis; automated, prospective linking is currently being implemented.

The findings in this report are subject to at least two limitations. First, both NORS and CaliciNet collect data on norovirus outbreaks; because norovirus outbreaks are defined as two or more cases of illness with a common exposure, these results might not be generalizable to endemic norovirus illnesses. Second, variations in reporting practices among both 
TABLE 2. Reported genotype, transmission mode, and outbreak setting of norovirus outbreaks before and after implementation of NoroSTAT surveillance in five states* — United States, August 2009-July 2015

\begin{tabular}{|c|c|c|c|c|}
\hline \multirow[b]{2}{*}{ Variables reported } & \multicolumn{2}{|c|}{ Pre-NoroSTAT (August 2009-July 2012) } & \multicolumn{2}{|c|}{ Post-NoroSTAT (August 2012-July 2015) } \\
\hline & $\begin{array}{l}\text { NoroSTAT states } \\
\text { No. (\%) }\end{array}$ & $\begin{array}{l}\text { Other states } \\
\text { No. (\%) }\end{array}$ & $\begin{array}{l}\text { NoroSTAT states } \\
\text { No. }(\%)\end{array}$ & $\begin{array}{c}\text { Other states } \\
\text { No. (\%) }\end{array}$ \\
\hline Genotype $^{\dagger}$ & $657(100)$ & $1,885(100)$ & $1,077(100)$ & $2,174(100)$ \\
\hline Gll.4 Den Haag 2006 & $39(6)$ & $121(6)$ & $4(0.4)$ & $5(0.2)$ \\
\hline Gll.4 Osaka 2007 & $5(0.8)$ & $5(0.3)$ & 0 & 0 \\
\hline Gll.4 New Orleans 2009 & $357(54)$ & $1,233(65)$ & $24(2.2)$ & $54(2.5)$ \\
\hline Gll.4 Sydney 2012 & $4(0.6)$ & $22(1.2)$ & $657(61)$ & $1,474(68)$ \\
\hline Other GII & $155(23)$ & $347(18)$ & $226(21)$ & $319(15)$ \\
\hline All Gl & $97(15)$ & $157(8)$ & $166(15)$ & $322(15)$ \\
\hline Transmission mode ${ }^{\S}$ & $1,357(100)$ & $2,843(100)$ & $1,981(100)$ & $3,738(100)$ \\
\hline Person-to-person & $958(71)$ & $2,191(77)$ & $1,456(73)$ & $2,952(79)$ \\
\hline Foodborne & $236(17)$ & $515(18)$ & $296(15)$ & $550(15)$ \\
\hline Environmental & $7(0.5)$ & $9(0.3)$ & $7(0.4)$ & $12(0.3)$ \\
\hline Unknown & $156(11)$ & $128(4)$ & $222(11)$ & $224(6)$ \\
\hline Outbreak setting ${ }^{\dagger}$ & $995(100)$ & $1,129(100)$ & $1,674(100)$ & $2,330(100)$ \\
\hline Long term care facility & $818(82)$ & $856(76)$ & $1,183(71)$ & $1,728(74)$ \\
\hline Restaurant & $19(2)$ & $18(2)$ & $59(4)$ & $59(3)$ \\
\hline Hospital/Other health care setting & $41(4)$ & $89(8)$ & $69(4)$ & $116(5)$ \\
\hline Child care center & $21(2)$ & $22(2)$ & $57(3)$ & $47(2)$ \\
\hline Other & $96(10)$ & $144(13)$ & $306(18)$ & $380(16)$ \\
\hline
\end{tabular}

Abbreviation: NoroSTAT = Norovirus Sentinel Testing and Tracking

* NoroSTAT-participating states during 2009-2015 are Minnesota, Ohio, Oregon, Tennessee and Wisconsin. Washington, DC and Puerto Rico are included with other states.

† Norovirus genotypes as reported to CaliciNet.

$\S$ Transmission modes and outbreak settings as reported to National Outbreak Reporting System (NORS).

NoroSTAT and non-NoroSTAT states can affect the quality and internal comparability of surveillance data.

Despite these limitations, the NoroSTAT network was shown to be valuable in early identification and better characterization of norovirus outbreaks across the country. Near real-time surveillance data from NoroSTAT improved public health response and preparedness when the GII.4 Sydney variant emerged during 2012-2013. Early reporting of data from NoroSTAT states allowed a timely assessment showing no increase in norovirus outbreak activity in the United States associated with emergence of the GII.4 Sydney variant, in contrast to data from other countries (4). Similar strain-specific attribution analyses can be useful to rapidly detect the impact of the emergence of novel norovirus strains every few years.

The NoroSTAT network has built upon the initial success demonstrated by the first five states, expanding to seven states in August 2015 and to nine states in August 2016. The more rapid, complete, and integrated reporting by NoroSTAT-participating states demonstrates a key advancement in norovirus outbreak surveillance, providing near real-time monitoring of norovirus outbreak activity and emerging new strains.

\section{Acknowledgments}

State, territorial, and local health departments for providing data on norovirus outbreaks, especially to those states participating in NoroSTAT.

\section{Summary}

What is already known about this topic?

Norovirus is the most common cause of acute gastroenteritis in the United States. Norovirus outbreaks are reported to CDC by state and territorial health departments. Reporting lags and incomplete reporting have been limitations to norovirus outbreak surveillance systems.

What is added by this report?

The initial five sentinel states that participated in the NoroSTAT network (Minnesota, Ohio, Oregon, Tennessee, and Wisconsin) during the first 3 years reduced the median reporting interval from 22 days to 2 days for epidemiologic data, and from 21 days to 3 days for laboratory data. These states also had more complete reports that better linked epidemiologic and laboratory data.

What are the implications for public health practice?

The NorOSTAT network provides near real-time surveillance of norovirus outbreak activity and emerging new strains. Data collected by NoroSTAT-participating states are representative of national trends and can help inform public health response.

\footnotetext{
${ }^{1}$ Epidemic Intelligence Service, CDC; ${ }^{2}$ Division of Viral Diseases, National Center for Immunizations and Respiratory Diseases, CDC; ${ }^{3} \mathrm{Oak}$ Ridge Institute for Science and Education, Oak Ridge Associated Universities.

Corresponding author: Minesh Shah, yxi8@cdc.gov, 404-718-1122.
} 


\section{References}

1. Green KY. Caliciviridae: The noroviruses. In: Knipe DM, Cohen JI, Griffin DE, Lamb RA, Martin MA, Ranacniello VR, Roizman B, eds. Fields virology, 6th ed. Philadelphia, PA: Lippincott Williams \& Wilkins; 2013:582-608.

2. Zheng D-P, Widdowson M-A, Glass RI, Vinjé J. Molecular epidemiology of genogroup II-genotype 4 noroviruses in the United States between 1994 and 2006. J Clin Microbiol 2010;48:168-77. http://dx.doi. org/10.1128/JCM.01622-09

3. Vega E, Barclay L, Gregoricus N, Shirley SH, Lee D, Vinjé J. Genotypic and epidemiologic trends of norovirus outbreaks in the United States, 2009 to 2013. J Clin Microbiol 2014;52:147-55. http://dx.doi. org/10.1128/JCM.02680-13

4. Leshem E, Wikswo M, Barclay L, et al. Effects and clinical significance of GII.4 Sydney norovirus, United States, 2012-2013. Emerg Infect Dis 2013;19:1231-8. http://dx.doi.org/10.3201/eid1908.130458

5. Hall AJ, Curns AT, McDonald LC, Parashar UD, Lopman BA. The roles of Clostridium difficile and norovirus among gastroenteritisassociated deaths in the United States, 1999-2007. Clin Infect Dis 2012;55:216-23. http://dx.doi.org/10.1093/cid/cis386
6. Lopman BA, Hall AJ, Curns AT, Parashar UD. Increasing rates of gastroenteritis hospital discharges in US adults and the contribution of norovirus, 1996-2007. Clin Infect Dis 2011;52:466-74. http://dx.doi. org/10.1093/cid/ciq163

7. Gastańaduy PA, Hall AJ, Curns AT, Parashar UD, Lopman BA. Burden of norovirus gastroenteritis in the ambulatory setting-United States, 2001-2009. J Infect Dis 2013;207:1058-65. http://dx.doi.org/10.1093/ infdis/jis 942

8. Hall AJ, Wikswo ME, Manikonda K, Roberts VA, Yoder JS, Gould LH. Acute gastroenteritis surveillance through the National Outbreak Reporting System, United States. Emerg Infect Dis 2013;19:1305-9. http://dx.doi.org/10.3201/eid1908.130482

9. Vega E, Barclay L, Gregoricus N, Williams K, Lee D, Vinjé J. Novel surveillance network for norovirus gastroenteritis outbreaks, United States. Emerg Infect Dis 2011;17:1389-95.

10. National Center for Health Statistics. US Census populations with bridged race categories. Hyattsville, MD: US Department of Health and Human Services, CDC, National Center for Health Statistics; 2015. https://www.cdc.gov/nchs/nvss/bridged_race/data_documentation.htm 